\title{
Using Participatory Data Analysis to Understand Social Constraints and Opportunities of Electricity Demand-Shifting
}

\author{
J. Bourgeois, J. van der Linden, G. Kortuem and B A. Price \\ The Open University \\ Milton Keynes, UK \\ \{jacky.bourgeois, j.vanderlinden, g.kortuem, b.a.price\}@open.ac.uk
}

\author{
C. Rimmer \\ EON UK, Technology Centre \\ Nottingham, NG11 OEE, UK \\ christopher.rimmer@eon-uk.com
}

\begin{abstract}
This paper reports results from an empirical study that explored the potential of interactive electricity DemandShifting - a particular form of behavior change where electricity consumption is shifted towards times of the day when production is at its highest - in the context of residential solar electricity generation. We conducted an in-the-wild user study with eighteen households over a period of six months, combining electricity data from smart meters and smart plugs with in-depth interviews to explore laundry routines and washing machine usage for each household. The study highlights that, although washing machines can be considered to be "shiftable appliances", the specific ways in which each household goes about shifting varies considerably. Furthermore, there is a clear need for ICTs to support people by analyzing current context and future plans to automate electricity demand-shifting and synchronization of both laundry and other appliances. Such support will help them maximize the self-consumption of micro-generated electricity while balancing this with environmental concerns, financial benefits and day-today issues relating to convenience.
\end{abstract}

Index Terms - Microgeneration, demand-shifting, in-home study, energy management.

\section{INTRODUCTION}

Evidence is beginning to emerge that households with solar photovoltaic systems (solar PV) exhibit energy saving behaviors intended to maximize the use of local electricity and to minimize the use of imported grid electricity $[1,2]$. Based on his study, Keirstead [1] uses the term "double dividend" to refer to the fact that microgeneration produces both green energy but also gives rise to energy saving behavior. He also observes that some households with microgeneration engage in "demand-shifting" - a particular form of behavior change where electricity consumption is shifted towards times of the day when production is at its highest. Similar behavior was observed by Price et al [2] who found that owners of solar PV installations were keenly interested in shifting energy intensive activities. Householders try to estimate when their houses generate the largest amounts of electricity and move energy intensive household activities like washing clothes and dishes to these times. However, the complex relationship between local weather conditions and electricity generation, the presence of seasonal variations and the complexities of generation systems and feed-in tariffs, makes it a difficult task for end users to predict residential electricity generation.
In this paper we report on a qualitative user study involving 18 UK households with solar PV panels on their roof. The aim of the study is to understand the context and challenges of selfconsumption - defined as users consuming their own production - and how we should design technologies to support households to engage in electricity demand-shifting. We specifically focus on households' laundry routines, as a regular activity carried out by all households that involves a number of electrical appliances. Using a variety of sources of electricity data for each household, we invited people to reflect on the specific circumstances in their household surrounding interactive shifting practices. How do these shifting practices fit with the residents' everyday life? How can we further generalize from routines around laundry practices to other household activities? The overall aim of these conversations around washing machine usage was to gain insights into interactive shifting in real-life settings, including the opportunities, constraints and the type of support that residents require for these activities.

In the remainder of the paper we describe the background relationships between electricity microgeneration, consumption and demand-shifting before we describe the methodology and results of our study.

\section{BACKGROUND: DISTRIBUTED GENERATION AND SHIFTING}

\section{A. Residential Electricity Gap and Self-Consumption}

The amount of electricity a solar PV installation generates depends on many factors: the type, age and condition of the solar PV equipment, their size and orientation of solar PV panels, geographic location of the house, weather conditions (cloud cover, rain, fog), season and time of day. These parameters make the amount of generated electricity highly variable during the day and across the seasons. Furthermore, the prediction of solar electricity output using weather forecasts is still unreliable [3].

In the residential context, the generation of electricity and the consumption of electricity are not synchronized, resulting in what we define as an electricity gap: there are periods (such as in the evening) where consumption exceeds local electricity generation and where electricity needs to be imported from the grid; and there are periods with high solar radiation (typically around midday) when electricity generation surpasses 
consumption and locally generated electricity can be exported to the grid, even in regions with less than ideal solar radiation, such as the UK.

The concept of self-consumption refers to the consumption of the locally produced electricity. This process is beneficial for the environment because it reduces the amount of electricity that gets lost through transporting it from one place to another $\left(8 \%\right.$ in the $\left.\mathrm{UK}^{1}\right)$. It is also in the financial interests of the householders. Depending on the precise national energy policy in place, households usually save more by reducing the amount that they need to import from the grid, compared to how much they would gain by selling their surplus to the grid. That is, it is usually cheaper to reduce the importing of electricity from the grid. From the grid point of view, self-consumption erases households from the grid and reduces instability related to sun and wind variability. This decreases the cost of energy providers by avoiding to maintain other energy sources such as gas or coal power plant as backup.

\section{B. Approaches to Energy Demand-Shifting}

Energy demand-shifting generally refers to the idea of using an appliance during a "better" period of time, that is when energy is cheaper (e.g. low period of consumption) or greener (e.g. sunny period). For example, running appliances during a sunny afternoon using local microgenerated power instead of during the evening, allows households to reduce the environmental and financial costs by reducing import and export of electricity. Demand-shifting has been researched through a number of different approaches, though not exclusively with the aim of increasing self-consumption of microgenerated electricity.

Barker et al discuss how appliances such as fridges, freezers and HVAC systems, referred to as background appliances, have loads that can be shifted in order to smooth the overall consumption [4]. In particular, their SmartCap system aims to flatten peaks in consumption which are caused when several appliances all consume electricity at the same moment in time. SmartCap provides fine-grained scheduling of such regular loads to ensure that they do not overlap. However, these appliances cannot be idled for very long periods, such as for a whole night while waiting for the sun or the wind to come up and this solution can therefore not easily be adopted to the scenario of microgenerated electricity.

Dynamic Pricing and Demand Response (DR) mechanisms are designed to implement demand-shifting. In such a system, "smart appliances" are able to receive and understand a signal sent by the electricity provider indicating the electricity tariff. Haghighi and Krishnaswamy argue that with DR "control actions are solely implemented based on the supply conditions and are regardless of the consumer's current situation" [5 p. 147]. Furthermore, DR approaches focus on grid consumption management but do not aim to optimize the self-consumption of microgenerated electricity.

Another approach to demand-shifting is to look at the types of appliances that are suitable for such practices. Allerding and colleagues developed a taxonomy of appliance-types, which distinguishes between controllable and observable devices [6]. In the controllable category are the permanent services that run continuously (such as a deep-freezer) and timed services (e.g. a dishwasher). In the observable category they make a distinction between predictable services (e.g. a stove or hob that is always used before meal times) and unpredictable services (e.g. lighting which is dependent on the weather and personal preferences). A different taxonomy of appliances is identified by Zhu et al [7] and is based on three categories: non shiftable (e.g. watching live television), time-shiftable (e.g. a washing machine) and power-shiftable (e.g. a water heater, or electric vehicle charger). This categorization of devices highlights interesting possibilities but focuses on a different problem instead of aiming to minimize peak hourly loads, the user in a microgeneration setting is interested in how to maximize loads during solar generation time. While studies $[4,5,6,7]$ consider the fact that users may have preferences, the proposed technological approaches do not interact with the user. Instead, these technologies are designed to be transparent for the residents.

A more user-centric approach is taken by Costanza and colleagues [8]. They demonstrate how an agent- based system, can help households shift laundry routines. However, this project takes place in the context of grid peak load reduction without considering self-generated electricity.

\section{Demand-Shifting and Solar-Powered Homes}

A number of studies have investigated the practice of demand-shifting in the setting of homes with solar PV. In the Netherlands, a study by Derijcke and Uitzinger [9] combined two surveys over 80 households - at the point when they have just bought solar PV and after 3 years - and the monitoring of overall consumption of four households. It highlights that participants shifted $15 \%$ of their washing machine, dishwasher and dryer loads from night to day times. Participants' motivation was more environmental than financial. Participants were using their generation meter to do this process manually.

In contrast, Banerjee and colleagues explored how home automation techniques can play a role in increasing selfconsumption [10]. Their study was based on the analysis of electricity consumption and generation data monitored in an off-grid house. They put forward three suggestions for tools to support householders: (i) an early warning to allow residents to anticipate a battery getting critically low, (ii) advice on the best time to execute high-power tasks and (iii) energy conservation suggestions such as refrigerator temperatures.

Kobus et al investigated how a washing machine that is able to wait for a sunny period (Smart Wash) impacts on the user [11]. Based on 21 interviews with householders the authors recommend that such technologies should integrate feedback with feed-forward information such as prediction, allowing the user to anticipate when the best time would be, but also a reward, such as green points or financial incentives as indicators of the "performance" and an acknowledgement of the effort this involves.

\footnotetext{
${ }^{1}$ Grid losses: http://data.worldbank.org/indicator/EG.ELC.LOSS.ZS/
} 
One way to address the issues raised by Kobus et al. is the use of Interactive energy Demand-Shifting (IDS) technologies, which we define as digital tools to engage the user in demandside electricity management to support intelligent electricity consumption - which reduces cost or environmental impact without necessarily reducing the consumption. IDS takes place through systems that make electricity and engagement with electricity more tangible. New electrical systems such as microgeneration and smart meters provide the first ingredient. Engagement comes through decision and motivation. Although householders now have increased access to electricity feedback, they still have to shift their consumption manually. In contrast with DR systems or transparent shifting such as SmartCap [4, 5], IDS takes input from users allowing them to both keep control and be aware of this electricity management. Our aim is to build on the insights of Kobus et al. by better understanding the decision making around laundry routines to uncover ICT support for IDS.

\section{USER STUDY}

We conducted a participatory user study with 18 households, over a period of 6 months. During this period residents carried out their normal laundry routines and we were able to track their electricity data through a variety of meters and smart plugs. The user study sits within a wider program of research, involving some 75 households investigating issues around household electricity usage. The 18 households we selected had all invested in solar electricity. From earlier focus groups and in-home visits we had become aware that participants had a keen interest in the amount of electricity they were generating and wanted to consume as much of it as possible. We learned that they manually shift some of their loads, like the washing machine or the dishwasher by "chasing the sunshine", that is, looking out of the window and switching on when it is sunny.

\section{A. Aim of the Study}

The aim of the study was to investigate more precisely how household members were carrying out this process of manually shifting their appliances. What were their struggles and constraints when aiming to maximize their self-consumption? How good were they at manually doing this, and what scope is there for further improvement? Underlying these questions was the further aim, to gain design insights for interactive demandshifting technologies that could support them further.

\section{B. Technology Set-Up}

Each of our participating households was equipped with three smart meters to measure: (i) imported electricity from the grid (the typical fiscal meter), (ii) generated electricity from the solar panels and (iii) the exported electricity to the grid. The smart meters recorded data every 3 minutes. Ten smart plugs were also deployed to monitor the electricity consumption of individual appliances. We were relying on the smart plugs to gain access to washing machine data. Apart from tracking the washing machine, households were free to monitor whichever appliances they were interested in. The participants were able to access their data via a web portal, a Smartphone and

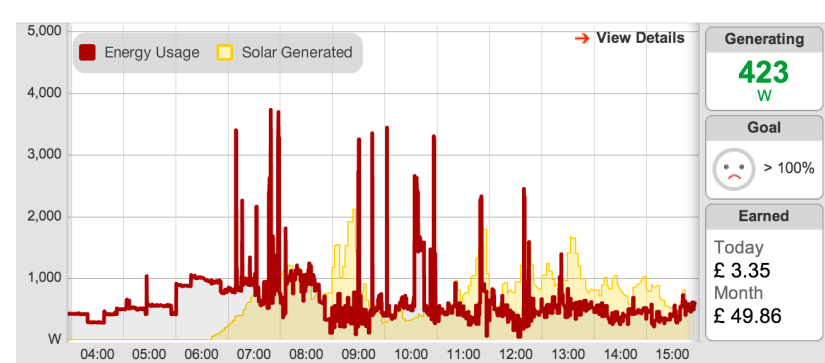

Figure 1: Web portal - Overall consumption (contrasted red curve) and generation (faded yellow curve)

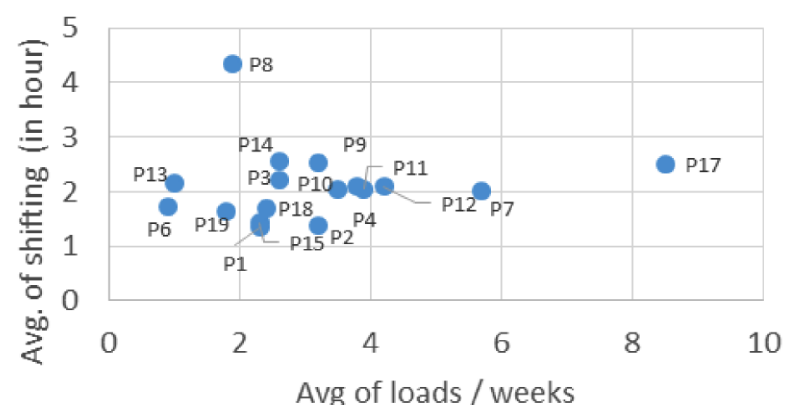

Figure 2: Distribution of the participants (average over 8 months)

\begin{tabular}{|c|c|c|c|c|}
\hline & People & $\begin{array}{l}\text { Occupation Home / work } \\
\text { balance }\end{array}$ & $\begin{array}{l}\text { Avg. loads } \\
/ \text { week }\end{array}$ & $\begin{array}{l}\text { W/M } \\
\text { location }\end{array}$ \\
\hline P1 & 2 adults & $\begin{array}{l}3 \mathrm{~d} \text { working away, } 2 \mathrm{~d} \\
\text { working from home }\end{array}$ & 2.3 & Kitchen \\
\hline $\mathrm{P} 2$ & 2 adults, 3 children & $\begin{array}{l}\text { Wife in on Mon, both out } \\
\text { Tues to Friday }\end{array}$ & 3.2 & Util. room \\
\hline P3 & 2 adults, 2 children & $\begin{array}{l}\text { Wife at home, husband } \\
\text { works mostly out }\end{array}$ & 2.6 & Util. room \\
\hline P4 & 2 adults, 2 children & Both working out & 3.8 & Kitchen \\
\hline P5 & 2 adults & Both working out & 0.9 & Kitchen \\
\hline P6 & $\begin{array}{l}2 \text { adults, } \\
1 \text { adult Mon-Fri, } \\
1 \text { child sometimes }\end{array}$ & About $50 \%$ in $/ 50 \%$ out & 5.7 & Util. room \\
\hline P7 & $\begin{array}{l}2 \text { adults, } 3 \text { children, } \\
\text { nanny }\end{array}$ & $\begin{array}{l}\text { Husband mostly away, wife } \\
\text { works, nanny at home } \\
\text { instead }\end{array}$ & 1.9 & Util. room \\
\hline P8 & 2 adults, 1 child & $\begin{array}{l}\text { Wife works at home, } \\
\text { husband works }\end{array}$ & 3.2 & Util. room \\
\hline P9 & 2 adults, 1 child & $\begin{array}{l}\text { Wife works at home, } \\
\text { husband works out }\end{array}$ & 3.5 & Kitchen \\
\hline P10 & 2 adults & Mostly at home & 3.9 & Util. room \\
\hline P11 & $\begin{array}{l}2 \text { adults, } 1 \text { child, } \\
1 \text { temporary guest }\end{array}$ & Work out & 4.2 & Util. room \\
\hline P12 & $\begin{array}{l}2 \text { adults, } \\
2 \text { children }\end{array}$ & Mostly at home & 1 & Util. room \\
\hline P13 & $\begin{array}{l}2 \text { adults, } 2 \text { children, } \\
2 \text { temporary guest }\end{array}$ & $\begin{array}{l}\text { Wife works at home, } \\
\text { husband works out }\end{array}$ & 2.6 & Util. room \\
\hline P14 & 2 adults & $\begin{array}{l}\text { Works from home, husband } \\
\text { works out }\end{array}$ & 2.3 & Util. room \\
\hline P15 & 2 adults, 2 children & Mostly at home & 2.4 & Kitchen \\
\hline P16 & 2 adults, 1 child & $\begin{array}{l}\text { Wife flexible shift and } \\
\text { husband 3-shift }\end{array}$ & 8.5 & Util. room \\
\hline P17 & 2 adults & $\begin{array}{l}\text { Retired, lot of activities } \\
\text { outside }\end{array}$ & 2.4 & Kitchen \\
\hline P18 & 5 adults & All working out & 1.8 & Util. room \\
\hline
\end{tabular}

Table 1: Participants 


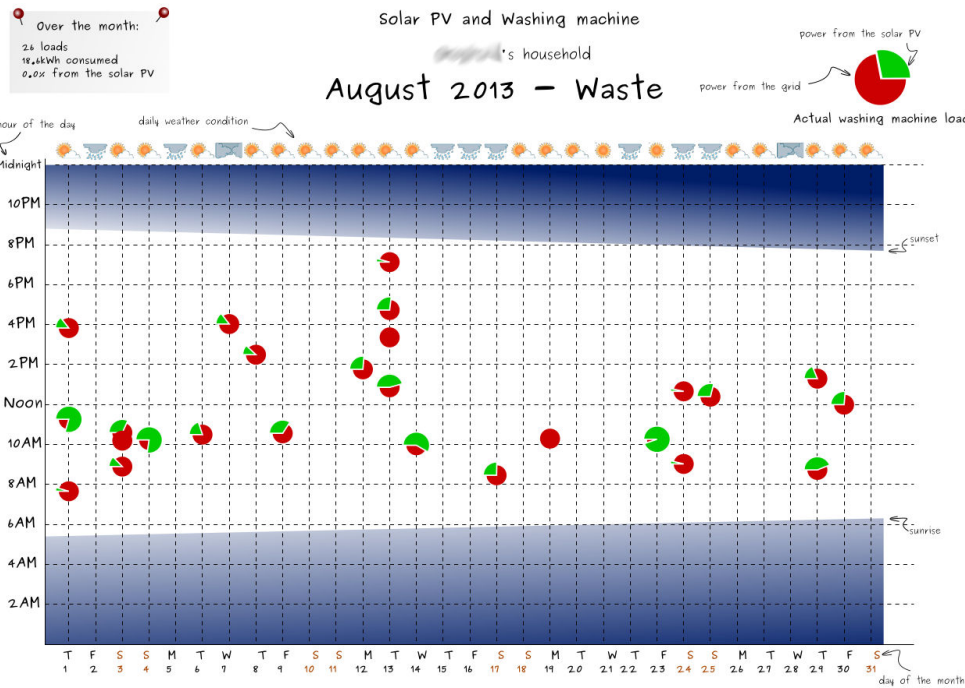

Figure 3: Visualization - Waste

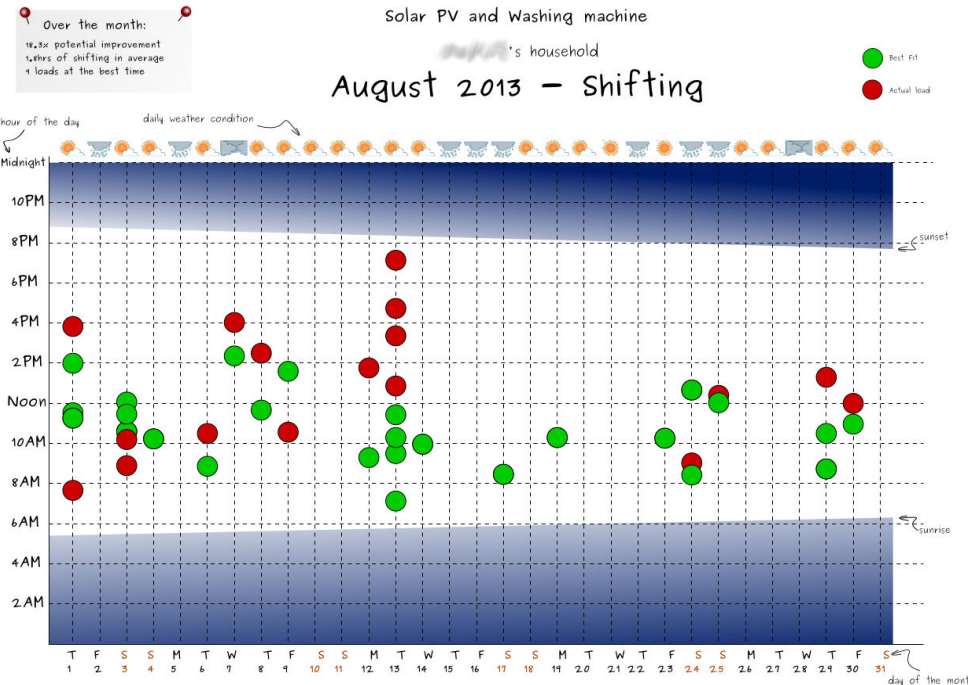

Figure 4: Visualization - Shifting

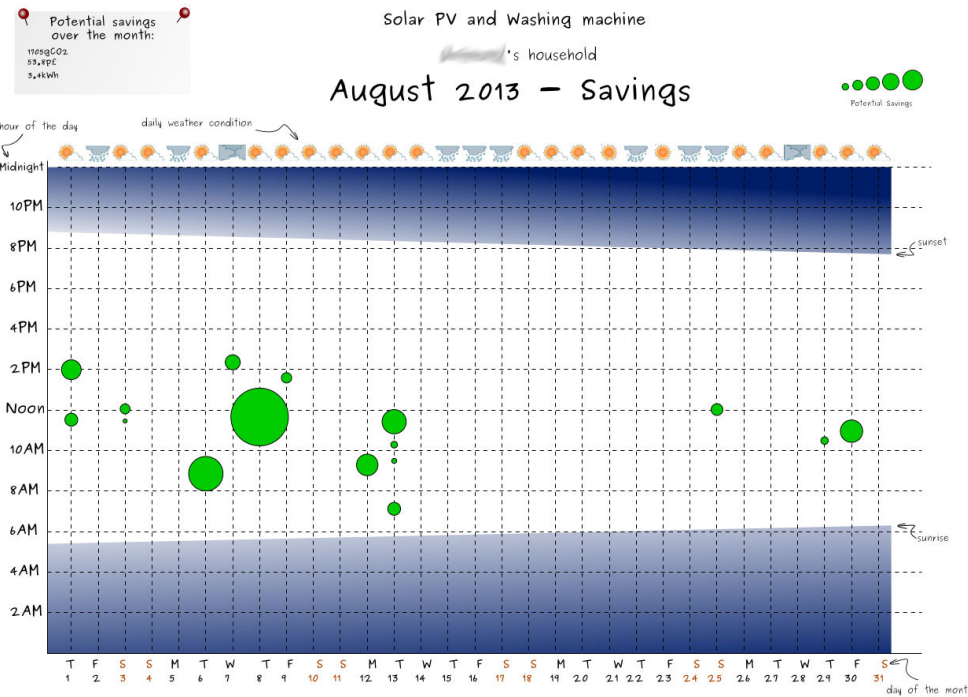

electronic tablet application allowing them to control smart plugs and to get instant and historical energy feedback (electricity, gas) on their consumption and generation (Figure 1). This data infrastructure had been developed over a period of almost two years and participants had a high awareness of home energy issues.

\section{Participants}

Although all participants owned their own homes they comprised a diverse demographic, from couples to large families, in employment or retired, spending most of their time at home or away. Table 1 details this diversity. Over half of the washing machines are installed in a separate utility room with the remainder being installed in the kitchen. The average load per week is calculated over the 6-month period of the study.

\section{METHODOLOGY}

Given the nature of the questions we wanted to answer through this study, it was important that the study was carried out as an in-the-wild study [12]. That is, we wanted households to carry out their washing routines in the naturalistic setting of their own families, with no specific instructions on what was required. At the beginning of the 6 month period households took delivery of their special washing machine and the smart plugs were connected to the machine.

\section{A. Analysis of Electricity Data}

Throughout the period electricity data from each household was analyzed to gain insight into common trends but also individual household patterns. We noted that households could be divided on two axes: (i) those who run a "lot of loads" against those who run "only few loads" per month (ii) households that are matching their local production with their energy consumption, and those who are less green. Figure 2 represents these two axes with (i) the average number of loads per weeks and (ii) the average time difference between the actual start time of their load and the optimal start time to maximize solar PV electricity use. In this study, we consider the "best users" to be those with an overall average start within 2 hours of the optimal time.

\section{B. Interviews}

At the end of the period, during two weeks in November and December 2013, we conducted interviews with each household. The aim of the interviews was to let residents reflect on their own laundry routines in the context of microgenerated electricity. In order to enable this process of reflecting we developed customized visualizations of people's personal electricity data. These interviews were conducted inhome lasting between 25 and 50 minutes and at a time suitable for the participants.

For each participant, we printed out a set of three visualizations for the most relevant summer month on A3 size paper. This way, participants were able to see clearly three different graphs at the same time, manipulating and comparing them easily on their table. We were also able to show further visualizations of data for other months on an electronic tablet which we could turn to if it seemed relevant in the context of

Figure 5: Visualization - Savings 
the conversation. After several interviews it became clear that the summer was too far back in time from the period of interview (end of November) for participants to remember specific events. We were then able to generate the same visualization for the month of November and show these on the electronic tablet. The whole idea of the paper and tablet-based visualizations was to give participants a way of looking at their own behavior and to talk us through their constraints and what affects their decisions.

\section{Visualizations}

The visualizations were developed to give participants an overview of their washing machine loads over a month. Each washing machine load was indicated as a distinct event in the week and month, and was represented as a multi-colored dot (Figures 3, 4 and 5). In these Figures the y-axis indicates which time the wash was started and the lower $\mathrm{x}$-axis indicates the day of the week and date for the wash. The sunrise and sunset times were visualized through dark blue bands, thus leaving a clear window of sunshine hours, shown to be gradually changing as the month progresses. The actual weather for each day, in the form of a "sunshine" or "cloud" symbol etc., was displayed at the top $\mathrm{x}$-axis providing contextual environment for each day.

The first visualization (Figure 3) uses a pie chart model for each load, showing for each load how much electricity was coming from the solar PV (lightly shaded part of circle = green in the original printed version) and how much electricity was coming from the grid (dark shaded part of circle $=$ red in the original printed version). The bottom of the pie chart represents the actual start of the load. For example, during the day on the far left this household did 3 lots of washing, one before 8 in the morning (using mostly grid electricity), one around noon (mostly electricity coming from solar energy) and one at 4 in the afternoon (again with mostly grid electricity). The participants were all very familiar with the concept of importing and exporting electricity and this first visualization was mainly designed to draw their attention to potential opportunities to increase their self-consumption. We deliberately gave this the title 'Waste' - to be provocative (even though there is no actual waste) and to make the point that participants could have consumed more electricity coming from solar energy and thus reduced their electricity import from the grid. The objective would be to have a full green circle, which means that the washing machine load had been entirely powered by the solar PV. This visualization thus gave a quick overview of the "green-ness" of the household's loads over the month and helped open the discussion.

The second visualization was designed to show participants when would have been the "greenest" time to start the washing machine and how much delay it would have implied. Figure 4 shows an example of this shifting visualization with the actual loads as dark circles (red in the original) and the best time for this load in light circles (green in the original). For example, in Figure 4, towards the middle of the month this household carried out four washing loads, during the afternoon (shown as dark circle), and as light circle is indicated that the morning would have been a better time for these loads, given the specific weather conditions for that day (see next section on how we calculated this). Using this chart the questions were phrased in terms of "Would it have been possible to..." with further questioning to find out the context and the constraints that made this happen: was it because they did not think about it? or was there a type of emergency that drove to these "bad" loads?

The final visualization is about savings: "How much will it save to shift a load". In Figure 5 the size of each circle represents the amount of savings; the position represents the best time to run the load. Many loads are invisible as the savings would be too small to represent in the diagram.

Note that shifting washing machine loads is often a matter of saving just a few pennies (dollar/euro cents) per month. However, this visualization was interesting because it allowed us to observe how participants, already aware of their own electricity, react to such tiny savings. How much do they care about such savings? How much effort are they prepared to invest in complex household activities to do with shifting? This visualization was also used to widen the discussion to other appliances and investigate whether such an electricity data analysis would be interesting for different appliances.

\section{Best Shifting Algorithm}

The visualizations made use of a best shifting algorithm to calculate the best fit in terms of shifting the load to a different time. In this study, a load represents one use of the washing machine. A load is characterized by the start time, the duration and the series of data points composed of a time stamp and a power value. The green percentage represents the amount of electricity $(\mathrm{kWh})$ used by a load that comes from the local solar panels compared to the total amount of electricity consumed by this load. Finally, the best fit of a load is the time slot that will provide the highest percentage of electricity coming from the solar panels during a specific day.

To determine the best fit of each load and generate the visualizations, we designed a system which analyses the electricity data of each day through three steps:

1. Synchronization: data are collected from heterogeneous sources and synchronized at a 1-minute sampling rate This includes the data coming from the import, export and generation meters and from the washing machine smart plug;

2. Load detection: the washing machine loads are detected and their details are stored. Then, these loads are removed from the overall consumption in order to isolate the consumption of the other appliances. It is then possible to add the washing machine loads to different points during the day to evaluate their impact;

3. Shifting: a brute force algorithm tries systematically every combination of loads over the day and computes a score based on a given objective.

In this study, our objective is to maximize the selfconsumption, i.e. to use as much electricity coming from the local solar panels. In other words, our goal is to minimize the overall export. The objective can be formalized as follows: 


$$
\text { minimize } E^{\prime}=I^{\prime}+G-\left(C_{\mathrm{WM}}+C_{R}\right)
$$

which represents over one day the potential export $\mathrm{E}^{\prime}$ given a potential import I', a generation $\mathrm{G}$, a washing machine consumption $\mathrm{C}_{\mathrm{WM}}$ and a remaining consumption $\mathrm{C}_{\mathrm{R}}$. It is important to highlight that this computation is based on actual import and actual export. However, after shifting we call these two variables "potential" (E' and I') because these are the values that we would have had if the washing machine load had been started at $t$. When there are several loads, the objective is to find the combination of best start times (i.e. best position of each load) that produces the best overall solution.

We implemented our system in Java and we sliced the analysis in 1-day periods. At this point the algorithm does not explore shifting towards a different day.

\section{E. Thematic Data Analysis}

In order to analyze the interviews, we followed the 6 phases of a thematic data analysis described by Braun and Clarke [13]. We fully transcribed the interviews and used Latex with the package ulqda [14] as a tool to code and extract the themes from the data.

\section{FINDINGS}

We extracted three main themes from our thematic data analysis: shifting and the context around shifting; decision making; and the issue of convenience. In this section we introduce general insights of the interviews then we go through these three main themes.

\section{A. Insights of Interviews and Visualizations}

There were some interesting trends we noted in the data. For example, households differed hugely in the number of washes they did per week, from less than 1 to more than 8 per week. It was not necessarily the case that the biggest households had the highest number of washes. Also, not many wash loads (only 315 out of a total of 1960 loads observed) were carried out during the dark hours when there is no generation.

Many participants expressed delight and surprise to see their own data presented through the visualizations. Many of them remarked that it was quite different from the views they usually see on the web portal, showing either real-time electricity data or historical trends (such as shown in Figure 1). P3-m reflects on the different types of insight gained from the visualization 1 .

P3- $m^{2}$ : "the portal actually gives us a summary of how much energy we use for the washing machine [...] This one now tells us that by adjusting the time, by shifting it (it makes no difference to us) so we could actually do that. But it's knowing when that best time is ..."

Many participants were also keen to better understand their own context at the time of the washing loads being visualized, saying: "can I just get my diary" (P11-w). With the help of their dairies they would bring up details, such as a busy week at work, school holiday, or visitors staying in the house which

\footnotetext{
${ }^{2}-\mathrm{m}$ for man and $-\mathrm{w}$ for woman
}

would further explain the pattern of washing loads they were looking at. One person had made specific notes about her washing loads herself, and was eager to compare these with the data presented in the graph - See Figure 6.

\section{B. Shifting in Context}

In our questioning we wanted to know whether participants could imagine doing even more shifting than they were doing already. That is, was there room for improvement? The few participants, who were already doing their washing at a very good point in the day, mentioned that seeing the visualization came as confirmation of how well they were doing. They did not think it would be possible to improve their behavior even further, but the insight confirmed that they were doing well and made them feel good. Others mentioned how the shifting visualization (Figure 2) could be interpreted as a target that they should aim to achieve. Some participants mentioned explicitly that they expected to be able to reduce the time between the actual start time and the best start time with the support of an automatic system.

\section{P16-w: "haha! so now I think I hope that the next time}

we see a chart like this that maybe there won't be so

much green on it because the weather is different. No,

but I hope we will be hitting the green marks"

The main objective of the "shifting" visualization was to understand if the participants would have been able to shift their actual washing machine load to the "best time". Many participants remarked that indeed a large number of loads were shiftable, and that if there were an automatic system to help them achieve that they would welcome it:

P16-w: "oh absolutely, if the machine is going to switch on then I can just pop it in the machine at 8 o'clock and it would run automatically at noon. So I come home and it's done"

P9-w: "I think that's certainly feasible to do, let say a load a day, and just fix it at the best time each day. That's not a problem"

With respect to being able to find this "best time" manually, for many people this appears difficult to achieve. This was even the case for those who spend a lot of time at home, and who consider themselves expert at catching the sunny time slots. This became evident around the "shifting" visualization by looking at the distance between the actual and the best start time which often showed a difference of 1 hour. Clearly some of the participants were disappointed, as they were expecting to see hundred percent green loads. It is interesting to observe this

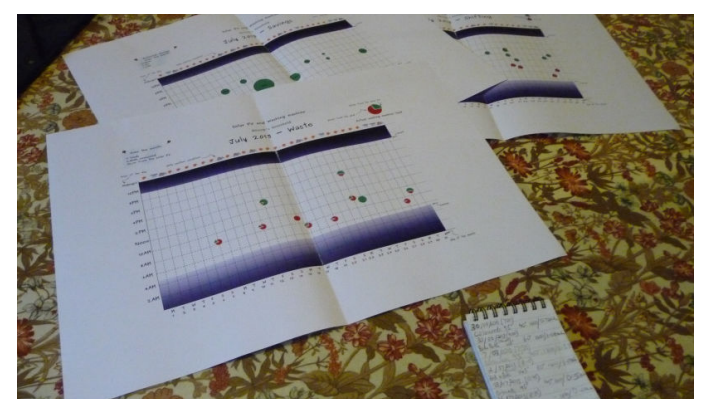

Figure 6: Interview setting with a participant pad containing the record of each washing machine load 
contradiction: the 'green' participants feel reassured by the "waste" visualizations, as it confirms they are on the right track - but the visualizations showing details of better starting times shows that they have still quite some scope for improvement.

\section{1) In and Out}

In the discussions around shifting, participants brought up a range of issues that stood in the way of them achieving the maximum self-consumption. One major recurring theme was work, and being away from home.

P7-m: "Me, I get up at 6 o'clock or 5:30. I'm out the door at about 6 and I'm back at 10 and I'm working all day. I don't really have much time to do anything, even at work. Let alone check this tab and the other. It's not that I don't think it's important but for me, me as a user..."

People discuss how they have different ways to cope with how they do their laundry: starting the washing machine or make sure it is finished by the time they leave the house, set up the delay on the washing machine to start later, or group the loads on a rest day or weekend.

Certain activities, such as school and sport, are also mentioned as two major obstacles to the washing machine load shifting. Many of the families with school age children mentioned it was imperative to wash the school uniforms over the weekend, to get them ready for Monday morning. They felt this was a process that could not be started before Friday evening, and for them the window of time to do the washing, drying and ironing did not offer a lot of flexibility.

P8-m: "he's at school (son), obviously. So we need to make sure about the end of the week that everything gets washed over the weekend, so it has a chance to dry. We don't use the tumble dryer - we dry naturally - so we try get it done. Although we would try using the washing machine during the day when we can, there are times when we run it at night or during the evening to make sure the wash loads are done."

Gym, tennis or other sports also pose various forms of constraints. These activities are often in the evening and a number of participants mentioned that they want to wash their clothes right away when they come back.

P8-m: "when my son comes back from tennis, all his tennis clothes go straight into the washing machine hmm..."

P1-w1: "I went to the gym, that was in the afternoon, I came back and washed the clothes [...] I don't like having smelly gym clothes lying around"

Participant P1 considers herself a very keen and green demand-shifter, with strict self-imposed rules to try and maximize the use of generated electricity. However, all her normal routines fly out of window when it comes to gym clothes - those have to be washed straight away.

A series of life events are often the source of a less controllable period. Families with younger children often talked of unpredictable emergencies such as a child being sick in their bed which then can lead to a different pattern of washing.

Older children who have left home but come back for longer periods insert a large amount of randomness in two different ways: first, they keep the parent busy and increase the number of dishwasher loads for example, or on a longer period those of the washing machine. Second, if they are using the washing machine they are not fully aware or concerned about using electricity from the solar panels.

P6-m: (talking about his daughter) "suddenly -oh I'm going back to college tomorrow - (gestures) - and then she puts everything in the washing machine..."

P5-w: "There have been couple of incidents when my son is here and I say (son's name), can you put it in the machine at a sunny time of day and he hasn 't...! He'd turned it on at 2 o'clock in the afternoon, which is not good for our solar!"

\section{2) Laundry}

The laundry activity itself also has internal constraints related to the demand-shifting process. A large number of participants use the term "wanting to clear the washing" or "catching up with the washing" expressing a way of doing their washing all at once, usually at the end of the week and over the weekend.

The particular design of the washing machine used by all participants also plays a significant role in the process of doing the laundry. First, the large drum of this washing machine allows the condensing of two loads into one. This reduces the number of washing loads that need to be done. Second, most of the program cycles take between two and three hours to run, as a result of the machine being designed to avoid large sharp peaks in its consumption of electricity. However, the length of the cycles reduces the flexibility of use. Some participants don't want to leave wet clothes sitting in the machine for a long time after the load completes, others are less concerned. Whether or not the user wants to be at home when the washing machine has just finished has an impact on the laundry time flexibility.

The method of drying is a direct constraint on the washing routine. Most participants do not use a tumble dryer because they are conscious that it is a high energy consumer.

For the participants who do use a tumble dryer, they do not use it systematically.

P16-w: "we don't use it through the summer, we use it sparingly in the winter"

For the participants who dry their clothes "naturally", there is a major constraint of time.

P8-m: "we do have constraints about when we need the washing dry by, because we don't have a tumble dryer we don't - we just dry the clothes"

Participants talk about having to find a balance between using the sun as electricity or as direct energy for drying. For many it is about running the washing machine early in the day in order to be also able to use the sun for drying the clothes naturally or for running the tumble dryer.

This model tends to be less constrained over the winter when drying clothes outside is not an option any more. 
P13-m: "Now in winter it's not much an issue because I don't dry outside so it doesn't really make much difference. So to be honest I can wash it at any time and the best time, as long as I'm around to take the washing out"

\section{Decision Making Processes}

One theme that appears as crucial in the context of laundry and electricity demand-shifting is the decision process. How do people decide? How do they make the choice to turn on the washing machine at a specific time? Is that decision really conscious? What are the motivations and parameters that play a role in this process? In most cases the decision relies on interpretation of information but also on expectations.

\section{1) Source of Information}

Most householders with solar PV on their roof relate their own electricity generation to the weather. Many of the participants use their smartphones or electronic tablets to check the weather forecast before starting a wash. Although this information is used either for the current and the following days, it is mostly used to determine whether the following days will be better (sunnier) or not. In other words, is it better to do the washing today or is it better to wait a couple of days? This 'shifting' decision comes into play when there are no specific constraints or emergencies for the current day.

P12-m: "I was looking at the weather. I go into the

tablet and look at the weather, think $O K$, but that's a

conscious decision by me to kind of do that and think

ahead."

Some of the participants, particularly older participants, do not find the weather forecast reliable enough generally. Even though most of them check the weather forecast - on their smartphones, tablets or TVs - they use what one participant call the "human element" while other participants talk about "looking out the window".

P11-m: "I don't find that the weather forecast can be reliable enough, certainly this time of the year when it does vary"

\section{2) Technical Considerations}

Participants also base their decisions on their expectations. Technical considerations can drive people to expect more generation in the morning because of the solar panel position on the roof for example. However, these expectations are sometimes wrong.

P5-m: “we've got more panels going south west than

we have south east so we would get more generation earlier on"

Some people were really surprised when being shown the washing machine loads visualization because they were expecting a different best time. One participant was not expecting such strong impact of the weather, thinking that running the washing machine at midday was obviously the best time.

P5-m: "yeah we put it on at half ten and you said the best fit was 2 o'clock, I would assume that half ten would have been a better fit than 2 o'clock".

\section{Motivations and Convenience}

Although the weather and other sources of information are used for a specific day or load, there are some more high level drivers such as saving money and taking care of the environment. In the UK those with both environmental impact and monetary savings goals find the situation more complicated, especially in winter. They need to decide between running more appliances during the night (when electricity is cheaper and a higher percentage comes from wind) or during the few sunny hours (when they can at least partly use local microgeneration).

Although most of the participants are convinced by the benefits of electricity self-consumption some were not convinced, even though they were still very much motivated by environmental concerns. For them exporting to the grid is equally about contributing to a greener energy system.

P16-w: “... but we import it again! So we probably export all this energy and then we buy it back, don't we? To run something else and that's surely not right." P17-w: “... as far as I'm concerned, as environmentalists, we are kind of happy contributing anyhow because it's not actually going to waste. It's being caught and used somewhere else. So I can't see the advantage of using energy directly compared to when it's going out of the house"

Money savings are generally strong motivations to drive the behavior. However, a balance has to be found between convenience and money savings. In fact, washing machines in general are not huge electricity users, especially the $\mathrm{A}+++$ energy rated washing machine we used for the study. On top of that, most of our participants were already trying to shift their load manually, which makes the potential savings even smaller. The average savings that our participants could have achieved over a summer month, i.e. if they had run all their actual loads at the best time, would have been about $£ 0.70(€ 0.85 / \$ 1.15)$ per month.

P6-m: “you've got two things going in parallel: the first thing is your life, you know. Certain things have to be done and start anyway so you can't concentrate 100\% on making sure that this is absolutely right; but in parallel with that you're also learning, this optimal period in the middle of the day, even if the sunshine is at 6:30 in the morning it's obviously not generating enough to do much with, hmm, so that is a sort of learning curve."

It was interesting to observe the reaction of participants looking at these monthly savings. Three of them thought instinctively of coffee: "3 pounds a year I mean! I buy a cup of coffee for that!" (P14-w). Participants think about the effort that is involved in shifting and how this balances with their comfort and convenience. Although they were mostly aware that they were not saving lot of money from the washing machine load shifting, they were all surprised to learn how tiny the benefits were. 


\section{E. Appliances}

\section{1) Shiftable/Non-Shiftable Appliances}

Extending the discussion from the washing machine to other appliances in the house, we built a map of the potential shiftable and non-shiftable appliances

The washing machine, dishwasher and dryer are the most common appliances that participants mentioned as shiftable. However, this topic was discussed in the context of interactive demand-shifting. However, there are only tiny savings to be made and participants were interested to discuss how they could approach demand-shifting with different appliances that they know as more important in terms of their consumption.

The hot water heater and space heating were mentioned as good shiftable devices. Participants described how to make demand-shifting much more automatic for these kinds of appliances. When automation would be expensive, e.g. replacing the device, participants suggested semi-automatic solutions. For example, P11-w suggested that she would be happy to receive a prompt by text message suggesting a good time to start the immersion heater (for the hot water cylinder). She felt she needed more information to know when it would be valuable to turn on the electric hot water instead of consuming gas to heat the water. All the participants but one have gas space heating, the most important part of the electricity bill. Although the solar panels cannot power the gas heating system, several participants describe situations where they have or they could have implemented an electric heating system in the living room to complement the gas consumption when they have spare electricity generation.

Other heavy and shiftable appliances are less common and participants discussed methods they have developed to manage them. For example, P2-m is the only one with a heat pump. In the summer, the device runs only to heat the water. He has set up a timer to turn it on only between $12 \mathrm{AM}$ and $2 \mathrm{PM}$ so that the pump gets powered through the solar panels. Other participants operate similar processes with appliances such a hot tub or their bread maker.

Participants also mentioned a list a non-shiftable devices including media devices and specifically the television. The oven is a heavy load and participants are well aware of the fact that it is a heavy consuming appliance. However, although they do not tend to change their dinner time, we did observe subtle changes in routines and habits around the usage of cooking appliances. For example, they were able to group the cooking of several dishes together, or aim to do most of their cooking during the day time, in advance, and for several days. They also mentioned avoiding the running of other major appliances at the same time as doing their cooking.

2) Interaction between Appliances

Most of the participants mentioned that there are issues to do with the interaction between appliances. As mentioned above, activities such as cooking (using the oven), cleaning (vacuuming, ironing) or more exotic devices such as a heat pump or hot tub have an effect on whether or not to run other appliances. Numerous participants mentioned they had developed a "rule" - which was "do not run at the same time".
P2-m: "I have the hot water on between 1 and 3 o'clock so we try not to run anything else between 1 and 3 $o$ 'clock because then we maximize. We never put the washing machine on around 2 o'clock I think"

Participants also mentioned that it is difficult to actually carry out this rule manually - to shift between the various appliances in the house. It can also lead to tension between members:

P6-m: "I say to her, well the tumble dryer is using a lot more energy so sometimes she says ok the sun is shining brightly, use the tumble dryer when that's finished to use the washing machine."

We can observe room for improvement and automation in this decision process.

\section{DISCUSSION}

The decision process around demand-shifting is time consuming and requires a combination of indicators ranging from the weather forecast to the "human element". This decision process is described as difficult for some participants lacking information or impossible for some others because they spend most of their time away. Similar to the conclusion of Banerjee in their study of an off-grid house [10], we highlight that participants want to anticipate: they base their decisions on real time or forecast parameters rather than energy feedback.

Previous research has highlighted a number of categorizations to indicate whether appliances are considered shiftable or not $[5,7]$. In these categorizations the emphasis is on the appliance itself and its functionality. However, our study has shown that there is wide variety in the ways different households engage with demand-shifting for one particular appliance - the washing machine. The washing machine is generally considered an appliance that is capable of being shifted with relative ease - but our research showed that for residents to properly engage with demand-shifting of the laundry is a considerable effort, and one that does not bear a huge economic benefit. The property of being shiftable does not so much depend on the appliance, but rather on the household, the residents and the specific situation. Furthermore, the specific design of the washing machine - its drum size, the length of its program cycles has a large effect on making it more or less shiftable. In particular, if considered in the context of residential electricity generation, a longer program cycle makes the appliance less flexible in terms of shifting, whereas shorter cycles make for easier scheduling. Furthermore, an appliance that is generally considered not to be shiftable, the cooker was discussed and we highlighted subtle ways for household members to change their routines around such appliances thus making the appliance more shiftable.

However, most of the participants clearly state that they are not able to run their washing machine at a better time without support. In emergency situations, there is needs for ICTs to select the least bad solution that allows users to satisfy a short term need. Apart from emergencies and being away, the biggest constraint to shifting appliance loads is related to the other appliances. Demand-Shifting is also about how to synchronize local electricity generation and multiple electricity 
consumption constraints. We highlight a need for ICTs to support this through a communication between appliances and user interfaces that distil information at the right time and adapt to the user to support the user in the decision process.

Finally, depending on the effort that the participants spend on increasing their self-consumption, we observed that the visualizations had different effects. While some visualizations could be used to help formulate targets, others act more as an acknowledgement - but the three visualizations together provided a vehicle for in-depth reflections on behavior and opportunities for householders to learn from this. We suggest that this information should be designed as a function of the motivation intensity of the participant.

This study goes beyond Kobus' work [11], highlighting the context and decision making around shiftable appliances and the strong relation between the different appliances in the house. It also confirms from the user point of view that there are shiftable and not-shiftable devices. However, most of them reduce the flexibility of each other's loads.

In-the-wild studies in the residential environment are challenging. This is even more difficult when it is about analyzing data from multiple electricity meters to compute detailed mappings of washing machine usage. However, our methodology - combining data analysis and in-home interviews - to discuss how to improve the self-consumption of a highly contextual activity such as laundry routines, revealed new insights and allowed participants to reflect and learn about their own behavior.

\section{CONCLUSION}

In this paper we reported on a study involving eighteen UK households with solar PV panels on their roof. The aim of the study was to understand the context and challenges of selfconsumption and how we should design technologies to support Interactive energy Demand-Shifting (IDS). We designed washing machine load visualizations to support demand-shifting and discussed them with each of the participants. We highlight the complexity of the environment and decision processes around demand-shifting and discuss the potential shiftable devices, noting that interaction and synchronization between appliances is a major obstacle to interactive demand-shifting and has to be part of further explorations. Through this paper we brought out the engagement and the willingness to engage in demand shifting. However, this process is time consuming and requires the consideration of many parameters from energy and weather predictions to contextual information. Users have access to increasing but still limited information and shifting happens manually. The questions remains: how to reduce the effort without reducing engagement? Further investigations should explore the potential of digital tools to address the highlighted challenges: which systems can support users by balancing convenience with the complex decision processes needed to deal with changing day to day constraints and priorities in electricity needs?

\section{ACKNOWLEDGEMENTS}

This study was carried out as part of the Thinking Energy project conducted by EON, a trial in a sample of 75 homes in Milton Keynes (UK). We wish to thank all the participating households as well as Simon Church, Chris Utting and Elizabeth Andrew for their support.

\section{REFERENCES}

[1] Keirstead, J. "Behavioural responses to photovoltaic systems in the UK domestic sector," Energy Policy, 35/8, Aug. 2007.

[2] Price, B. van der Linden, J., Bourgeois, J. and Kortuem, G.. "When looking out of the window is not enough: informing the design of in-home technologies for domestic energy microgeneration." In: First International Conference on Information and Communication Technologies for Sustainability, 14-16 February 2013, Zurich, Switzerland.

[3] Lorenz, E., Hurka, J., Heinemann, D. and Beyer, H. G. "Irradiance Forecasting for the Power Prediction of GridConnected Photovoltaic Systems.” IEEE Journal of Selected Topics in Applied Earth Observations and Remote Sensing 2, no. 1 (March 2009): 2-10.

[4] Barker, S., Mishra, A., Irwin, D., Shenoy, P. and Albrecht, J. "SmartCap: Flattening peak electricity demand in smart homes." PerCom. 2012. 67--75.

[5] Haghighi, P. D. and Krishnaswamy, S. "Role of contextawareness for demand response mechanisms." In Information and Communication on Technology for the Fight against Global Warming, 136-149. Springer, 2011.

[6] Allerding, F. and Schmeck, H. "Organic Smart Home: Architecture for Energy Management in Intelligent Buildings." In Proceedings of the 2011 Workshop on Organic Computing, 2011, 67-76.

[7] Zhu, Z., Tang, J., Lambotharan, S., Chin, W., and Fan, Z. "An integer linear programming based optimization for home demand-side management in smart grid." Innovative Smart Grid Technologies (ISGT), 2012 IEEE PES 2012. 1--5

[8] Costanza, E., Fischer, J. E., Colley, J. E., Rodden, T., Ramchurn, S. and Jennings, N. R. "Doing the Laundry with Agents: A Field Trial of a Future Smart Energy System in the Home". CHI 2014.

[9] Derijcke, E. and Uitzinger, J. "Residential Behavior in Sustainable Houses." In User Behavior and Technology Development, 119-126. Springer, 2006.

[10] Banerjee, N., Rollins, S., and Moran, K. Automating energy management in green homes. Proc. SIGCOMM HomeNets '11, ACM Press (2011), 19-24.

[11] Kobus, C. B., Mugge, R. and Schoormans, J. P. "Washing When the Sun Is Shining! How Users Interact with a Household Energy Management System." Ergonomics 56, no. 3 (March 2013): 451-462.

[12] Brush, A. J., Lee, B., Mahajan, R., Agarwal, S., Saroiu, S. and Dixon, C. "Home Automation in the Wild: Challenges and Opportunities." In Proceedings of the SIGCHI Conference on Human Factors in Computing Systems, 2115-2124. ACM, 2011.

[13] Braun, V. and Clarke, V. "Using Thematic Analysis in Psychology." Qualitative Research in Psychology 3, no. 2 (January 2006): 77-101.

[14] Griffin, I. and Richardson, I. "Using LATEX for Qualitative Data Analysis." The PracTEX Journal 1 (2010). 